\title{
Residuo liquido agroindustrial aplicado como enmienda en un suelo bajo un sistema de cultivo de maíz (Zea mays)
}

\author{
Milton Cesar Ararat Orozco ${ }^{1}$, Oscar Eduardo Sanclemente Reyes ${ }^{2}$, Carolina Hernández Rivera ${ }^{3}$ \\ ${ }^{1} \mathrm{PhD}$. Ciencias Agropecuarias, ${ }^{2} \mathrm{PhD}$. Agroecología, ${ }^{3}$ Ing. Ambiental. \\ ${ }^{1}$ Docente Escuela de Ciencias Agrícolas, Pecuarias y del Medio Ambiente, UNAD. \\ ${ }^{2}$ Líder Grupo de investigación Producción sostenible \\ 1․milton.ararat@unad.edu.co, ${ }^{2}$ oscar.sanclemente@unad.edu.co, ${ }^{3}$ caroh83@hotmail.com
}

\begin{abstract}
Resumen
Con el propósito ambiental de aprovechar una sustancia proteica obtenida de la agroindustria del maíz (molienda húmeda) denominada SUSTPROT, se aplicaron en forma de enmienda algunos tratamientos líquidos en un Inceptisol de la zona plana del municipio de Jamundí, Valle del Cauca, cuyo diseño experimental correspondió a BCAA y en un sistema de cultivo con un hibrido de maíz (Zea mays). Se analizaron variables en las propiedades químicas del suelo, con los siguientes tratamientos: $\mathrm{S}+\mathrm{M}$ (susprot + micelio), $\mathrm{S}+\mathrm{A}$ (susprot + agroplus), $\mathrm{S}$ (susptrot), $\mathrm{S}+\mathrm{M}+\mathrm{A}$ (susprot+micelio+agroplus) y T (Testigo: fertilización convencional). Los resultados indicaron diferencias estadísticas en las variables Fósforo, Boro. Se encontró asociación negativa entre el contenido de fósforo foliar y el del suelo $(r=0.92)$, indicando que el SUSTPROT, incremento significativamente la disponibilidad y absorción de dicho nutriente por parte de la planta. Con el tratamiento "S" se obtuvo mayor rendimiento $5.65 \mathrm{t} / \mathrm{ha}$.
\end{abstract}

Palabras clave: Fósforo; intercambio iónico; residuos agroindustriales del maíz; SUSTPROT.

\section{Introducción}

En Colombia, el Departamento Nacional de Planeación proyectó para el 2018, el 20\% en aprovechamiento de residuos que van a los rellenos e incentivar el reciclaje, sin embargo, no ha logrado superar el $17 \%$, por lo tanto, es prioritario incluir mecanismos de articulación del componente productivo y de investigación científica y tecnológica (Peñaranda et al., 2017).

La fertilización del cultivo de maíz (Zea mays) ha merecido gran número de estudios en el departamento del Valle del Cauca, sin embargo es pertinente hacer énfasis frente al proceso de perdida de la fertilidad de suelo y/o degradación, para los cuales se requieren medidas de manejo urgentes; en la industrialización de este cereal, durante el proceso de molienda húmeda, se generan subproductos de carácter orgánico-mineral que pueden aplicarse en forma de fertilizante y/o enmienda al suelo y tener efecto sobre las propiedades químicas y biológicas.

La aplicación de residuos orgánicos, es capaz de modificar algunas características del suelo tales como la $\mathrm{CIC}$ y el contenido de carbono orgánico. Estos cambios se traducen en un incremento de la productividad del suelo, la cual puede ser evaluada a través del rendimiento de cultivos (Sivapalan 1981). Las grandes cantidades de residuos agroindustriales, en este caso el SUST PROT obtenido de la molienda húmeda de maíz, puede tener una gran valorización agronómica y debe ser el objetivo prioritario, siempre y cuando se realicen los tratamientos necesarios para garantizar la calidad del producto y se programen los planes de aplicación a suelos con aptitud agrícola, contribuyendo a la disminución de los costos de producción.

Estas consideraciones con productos orgánicos pueden estar inmersas en una nueva visión ecológica en labores agrícolas, así como lo argumenta Sanclemente (2015) Corredor (2012), el cual da un contexto asociado a los mercados verdes donde el productor y consumidor generen un diálogo de saberes entorno a la importancia de consumir productos inocuos, dando valor agregado a la salud,

DOI: https://doi.org/10.22490/ECAPMA.2775 
al mismo tiempo que se asegura la soberanía alimentaria de la población local al reducir la importación de productos.

\section{Marco teórico}

Una característica muy particular de los fertilizantes o enmiendas de origen orgánico es que los nutrientes, a excepción del potasio, se localizan predominantemente en forma orgánica y por lo tanto en forma insoluble, en particular en los residuos sólidos. Sin embargo, aquellos presentes en los residuos líquidos están presentes en forma soluble; por lo tanto, para la absorción por las plantas deben transformarse a la forma inorgánica mediante la descomposición de la materia orgánica o mineralización. Así se produce una lenta liberación de nutrientes para la solución del suelo (Montaño, 2000).

No obstante, Los residuos provenientes de la actividad agroindustrial y que probablemente contengan fuentes orgánicas que se puedan transformar y en el suelo pueden contribuir al enriquecimiento de algunas propiedades como la materia orgánica (MO); según Burbano (1994), esta característica influye sobre las diversas propiedades del suelo, además posee propiedades físicas y químicas que facilitan la agregación, modifica la estructura física del suelo, e influye en los regímenes de agua; es una fuente de energía para la biota del suelo, por lo que influye en varios 'procesos que son movidos biológicamente.

Puede considerarse práctico algunos efectos de estos residuos orgánicos líquidos como el efecto sobre la retención de humedad, el cual contribuye al efecto positivo sobre la estructura mejorando el transporte y almacenamiento de agua, mejorando la infiltración, disminuyéndose las pérdidas por escorrentía y por evaporación directa. Por otra parte, la MO al reducir escorrentía, y favorecer la agregación lleva a menores riesgos de erosión, tanto hídrica como eólica (Kiehl, 1985).

Además de usarse como enmienda, los residuos líquidos podrían tener comportamiento similar a biofertilizantes a partir de compostaje, así como lo afirman Montenegro \& Ararat (2015), ya que son de gran utilidad en el acondicionamiento físico-químico del suelo, ligándose a sus partículas y contribuyendo principalmente en la retención de nutrientes y la aireación del mismo.

Así como lo afirma Sanclemente et al., (2018) y Amaringo (2013), desde un enfoque integral que incluye la generación, manejo e impacto ambiental, es pertinente asociar los resultados de estudio de caso con la elaboración de programas de gestión integral de residuos para su manejo adecuado y futuro aprovechamiento.

\section{Materiales y métodos}

El presente estudio se realizó en la finca La Novillera, municipio de Jamundi Valle del Cauca $\left(3^{\circ} 17^{\prime} 42.35 \mathrm{~N}-76^{\circ} 32^{\prime} 01.50 \mathrm{~W}\right)$, con un sistema tecnificado de maíz (Zea mays) (Híbrido Pionner 30k75); Los aspectos biofísicos del sistema de cultivo se midieron a través de la metodología sugerida por Ararat et al. (2014), a través de una matriz de datos con parámetros físicos y químicos para el reconocimiento del terreno. Se utilizó un residuo liquido proveniente de la molienda húmeda de tipo agroindustrial del grano de maíz denominada sustancia proteica (SUSTPROT), se aplicaron en forma de enmienda tratamientos líquidos en un Inceptisol de la zona plana del municipio de Jamundí, Valle del Cauca, se tomaron muestras de suelo y foliares al inicio y al final del ciclo del cultivo. Estas se llevaron al laboratorio para su correspondiente determinación. Para el análisis estadístico de resultados involucró la interpretación de los análisis de laboratorio y del rendimiento del cultivo. El diseño experimental fue Bloques completos al azar (BCAA) donde se estableció arreglos según la topografía del terreno de acuerdo a su ubicación relativa en la pendiente y cuyo esquema se compuso de 3 bloques con 5 tratamientos y 3 repeticiones por cada tratamiento distribuidos al azar; las respectivas parcelas con un área $100 \mathrm{~m}^{2}$ y sobre las que se han realizado siembras convencionales del maíz. El muestreo del suelo se realizó en dos (2) épocas, el primero un (1) día antes de la preparación del suelo para la siembra y el segundo un (1) día después de cosecha mecanizada a una profundidad de $20 \mathrm{~cm}$. En cuanto a parámetros relacionados con el nivel 
de fertilidad del suelo, se analizó la tendencia a través del tiempo de la capacidad de intercambio catiónico $\mathrm{CIC}$ con muestreos cada mes.

Los datos procesados estadísticamente con las pruebas de Duncan para determinar si se presentan diferencias significativas entre tratamientos, para las variables químicas del suelo y correlación de Pearson para determinar cuáles variables tienden a estar relacionadas entre sí, ya sea directa (positiva) o inversa (negativa). En la descripción de tratamientos (Tabla 1) se especifica la adición de otros productos con minerales microorganismos benéficos que posibilitan la aceleración de la descomposición y mineralización de los compuestos orgánicos:

Tabla 1: Descripción de tratamientos

\begin{tabular}{|c|l|l|}
\hline Tratamiento & \multicolumn{1}{|c|}{ Aplicación } & \multicolumn{1}{|c|}{ Especificaciones } \\
\hline T1 & susprot + Micelio & $\begin{array}{l}\text { Micelio: Residuo orgánico industrial } \\
\text { para la obtención de ácido cítrico }\end{array}$ \\
\hline T2 & susprot + Agroplus & $\begin{array}{l}\text { Agroplus: Bacterias nitro fijadoras, } \\
\text { lactobacilos, hongos y levaduras de } \\
\text { carácter comercial }\end{array}$ \\
\hline T3 & susptrot & \\
\hline T4 & susprot + Micelio + Agroplus & Insumos de síntesis química: NPK \\
\hline Testigo & Fertilización convencional &
\end{tabular}

Fuente: Autores

La aplicación de los productos en los tratamientos se realizó a los 45 DDS (Después de la siembra) por medio de un tractor equipado con un tanque y con sus respectivas boquillas de aspersión. La dosis se representa de la siguiente manera: SUST PROT: $1500 \mathrm{Kg}$. /ha, Micelio: $100 \mathrm{Kg}$. /ha y Agroplus: $130 \mathrm{~L} /$ ha. Según los requisitos específicos de la Norma Técnica Colombiana, este producto no aplicaría dentro de la clasificación como fertilizante o abono, pero si se aproxima a clasificarse como Enmienda Orgánica no Húmica siempre y cuando tenga un proceso especial de transformación y/o manejo. Uno de los principales requisitos que se cumple es el porcentaje de carbono orgánico que está por encima de la norma (mínimo 30\%). En los macro-elementos el alto contenido de nitrógeno, fósforo y potasio, como también niveles altos para todos los micro-elementos como el hierro en este caso (Tabla 2).

Tabla 2. Composición química del SUSPTROT

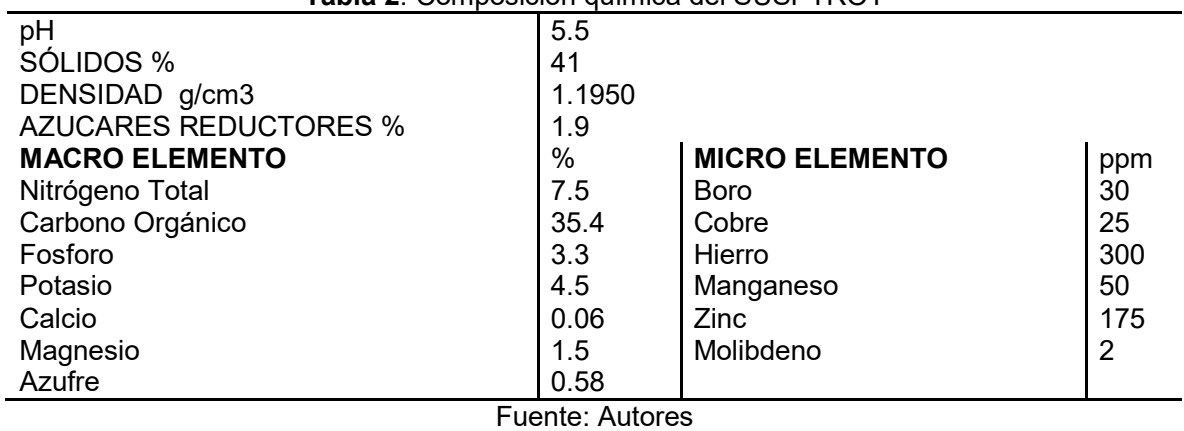

\section{Resultados y discusión}

El análisis de varianza muestra que existen diferencias significativas solamente en las variables Fósforo y Boro del suelo por efecto de tratamientos (Tabla 3). 
Tabla 3. Grupos LS Means (Media Mínima Cuadrática) para las variables de respuesta asociadas a propiedades químicas en la finca Novillera (Jamundi - Valle del Cauca).

\begin{tabular}{|c|c|c|c|c|c|c|}
\hline \multicolumn{3}{|c|}{ Fósforo (ppm) } & \multicolumn{4}{|c|}{ Boro (ppm) } \\
\hline Tratamiento & Promedio & $\begin{array}{l}\text { Media Minima } \\
\text { Cuadrática }\end{array}$ & Tratamiento & Promedio & $\begin{array}{r}\mathrm{Med} \\
\mathrm{Cu}\end{array}$ & $\begin{array}{l}\text { ima } \\
\text { ca }\end{array}$ \\
\hline TESTIGO & 36,12 & $a$ & TESTIGO & 0,25 & $a$ & \\
\hline $\mathrm{T} 4(\mathrm{~S}+\mathrm{M}+\mathrm{A})$ & 28,24 & a & $\mathrm{s}$ & 0,18 & a & $b$ \\
\hline $\mathrm{T} 2(\mathrm{~S}+\mathrm{A})$ & 27,24 & a & $S+M$ & 0,15 & a & b \\
\hline T3 (S) & 23,04 & $b$ & $S+M+A$ & 0,12 & & b \\
\hline $\mathrm{T} 1(\mathrm{~S}+\mathrm{M})$ & 22,86 & $b$ & $S+A$ & 0,11 & & $b$ \\
\hline
\end{tabular}

Fuente: Autores

El tratamiento testigo que tuvo la respectiva fertilización convencional fue el que presento mayor fósforo en el suelo después de la aplicación (figura 1), mientras que los tratamientos " $S$ " y " $S+M$ " presentaron menor contenido en el suelo, sin embargo, estos 2 últimos mostraron los mayores rendimientos en cosecha.

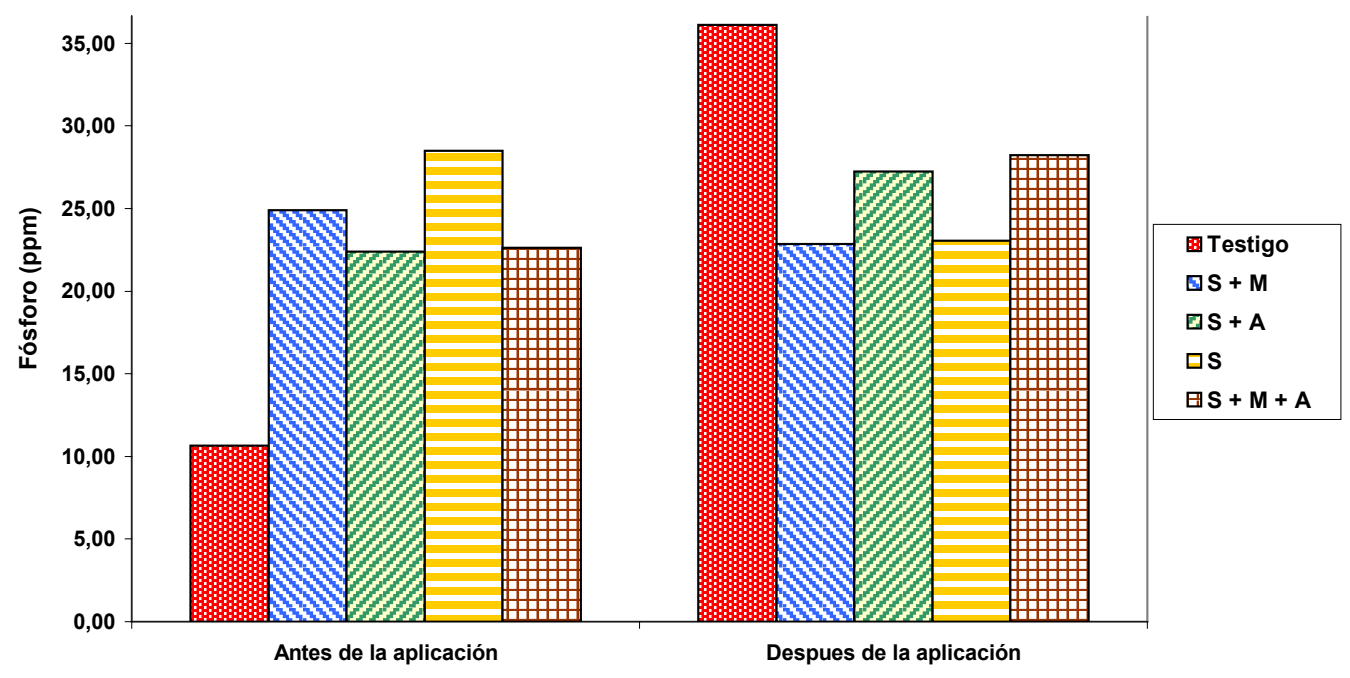

Figura 1. Efecto de tratamientos sobre la variación de fósforo edáfico en la finca Novillera - Jamundi. Fuente: Autores

En este suelo se correlacionó la variable fósforo del suelo con el análisis foliar donde el testigo resulto con menor contenido foliar (0.25\%) (Tabla 4 ) y mayor en el suelo; por el contrario, el tratamiento S+A resultó con mayor contenido de Fósforo en los tejidos y menor en el suelo (Figura 2); lo cual podría deberse a que durante el proceso de descomposición de este producto se producen algunos compuestos que estarían interactuando con las formas de fósforo presentes en este suelo y posiblemente se harían disponibles para la planta como consecuencia de esta interacción. 
Tabla 4. Análisis de Fósforo en el tejido Foliar de maíz (híbrido 30k75).

\begin{tabular}{c|c}
\hline Tratamiento & Fosforo Foliar (\%) \\
& \\
T1 $(\mathrm{S}+\mathrm{M})$ & 0,28 \\
T2 (S+A) & 0,35 \\
T3 (S) & 0,28 \\
T4 (S+A+M) & 0,31 \\
Testigo & 0,25 \\
\hline
\end{tabular}

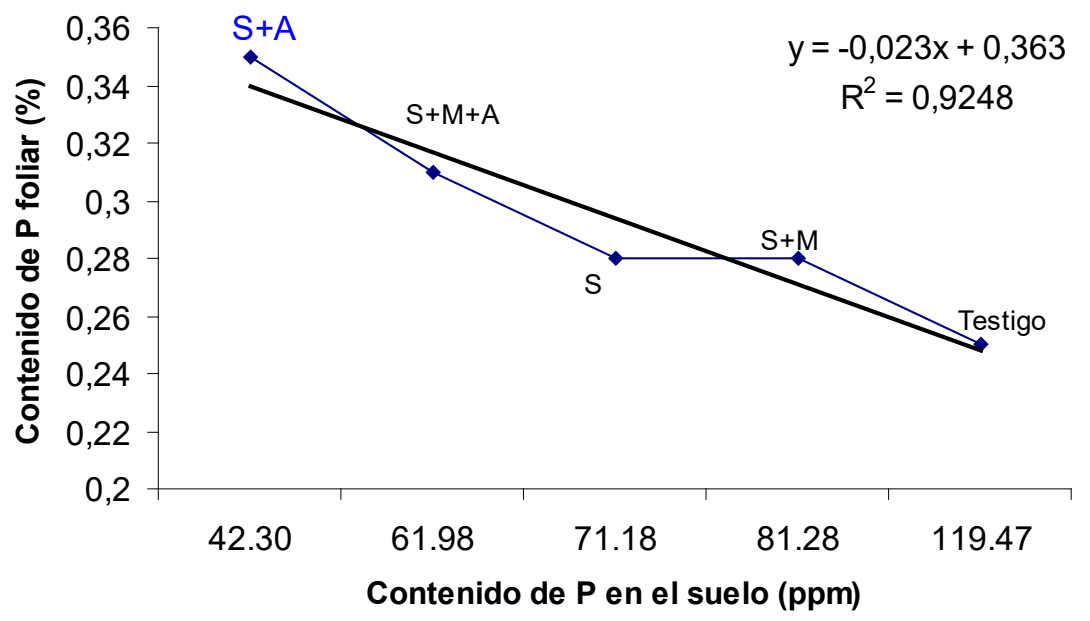

Figura 2. Relación entre el contenido de P en un hibrido de Maíz (30k75). Fuente: Autores

Estos resultados son coincidentes con lo señalado por Kiehl (1985), quien indica un efecto positivo de residuos de origen vegetal sobre la disponibilidad y absorción del Fósforo, bien por aporte directo vía degradación y mineralización rápida, bien por la interacción de productos de las primeras fases de degradación del Residuo orgánico y formas inorgánicas del fósforo presentes en el suelo.

De acuerdo a sus características taxonómicas del suelo, en la variable $\mathrm{Fe}^{+2}$ se destacan altos valores iniciales en el suelo, sin embargo, después la aplicación se presentó un incremento considerable (Figura 3), teniendo en cuenta los altos niveles de este elemento en el SUSPROT.

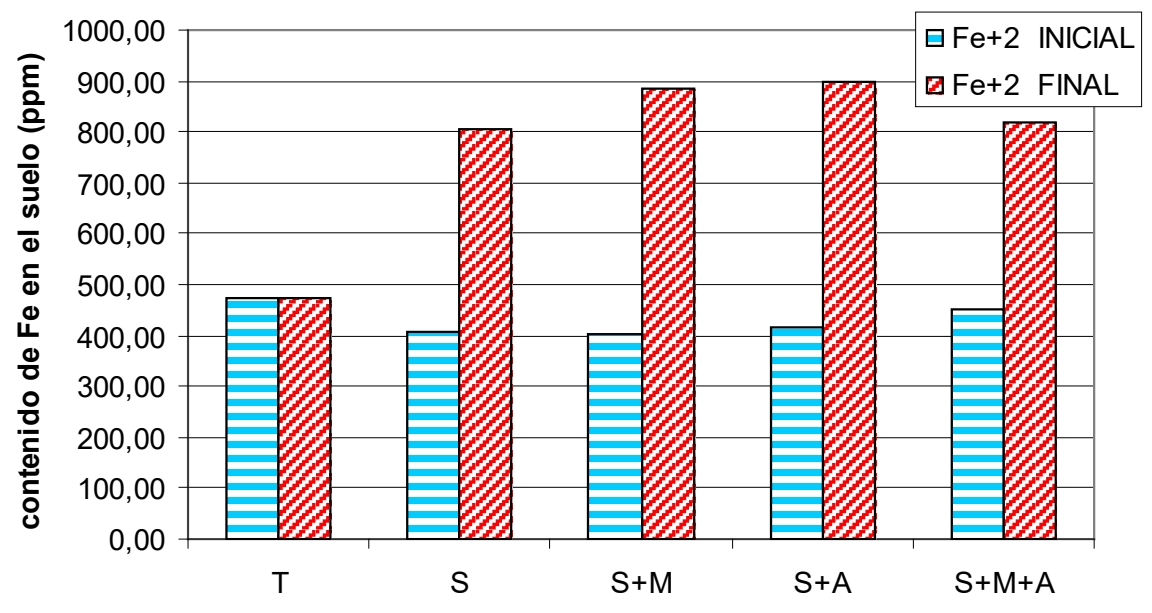


Figura 3. Contenidos de hierro $\left(\mathrm{Fe}^{+2}\right)$ de un Inceptisol en 2 épocas en la finca Novillera Jamundi (Valle). Fuente: Autores

Otra respuesta descriptiva en el suelo fue la Capacidad de Intercambio Catiónico (CIC) donde los máximos y mínimos estuvieron por encima de valores iniciales (Figura 4), lo que podría explicar el aporte de cargas de

radicales de los compuestos orgánicos al suelo.

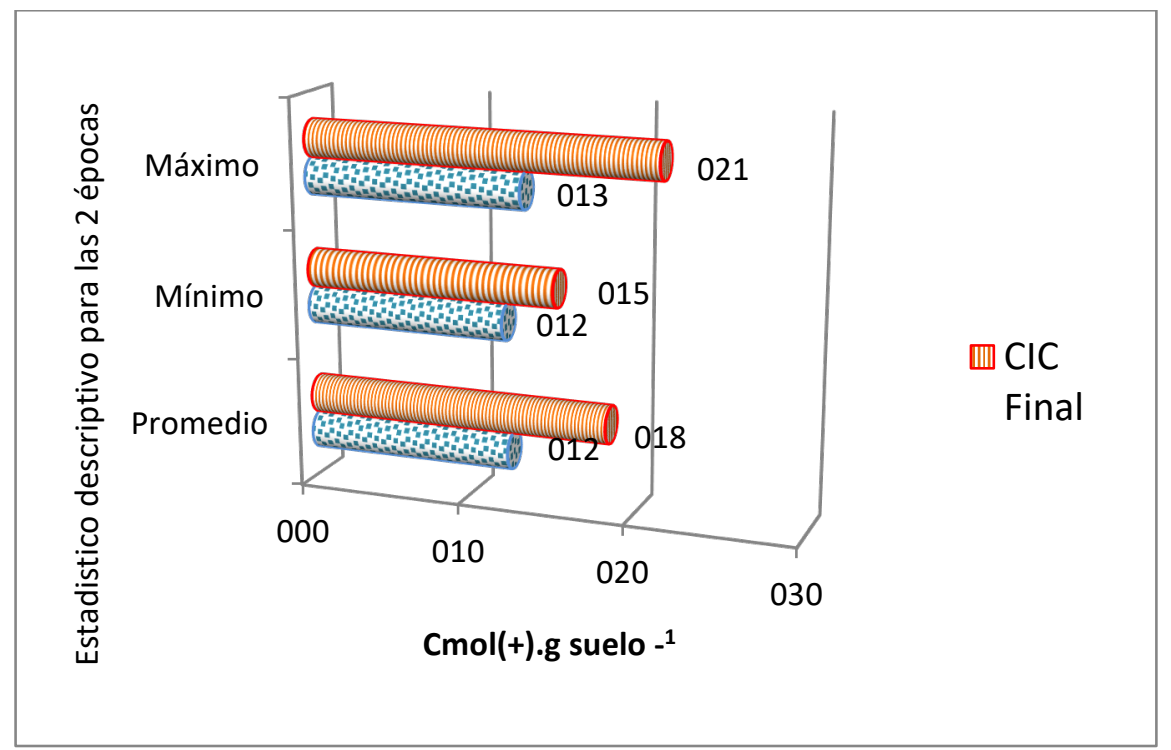

Figura 4. Aumento de la $\mathrm{CIC}\left(\mathrm{Cmol}^{+} . \mathrm{kg}^{-1}\right)$ del suelo en 2 épocas de muestreo. Fuente: Autores

Con relación a los tratamientos, con la aplicación de $S+M+A(T 4)$, se presentó el mayor incremento de los cationes intercambiables (Figura 5), es decir una variación con tendencia al aumento en todos los tratamientos con SUSPROT. Este efecto corresponde a la descomposición de los y su interacción con las fuentes de carbono de la materia orgánica residente en el suelo y otros materiales arcillosos; De acuerdo con Ryan \& Singh (2015), estos procesos regulan los ciclos de nutrientes síntesis de otros minerales.

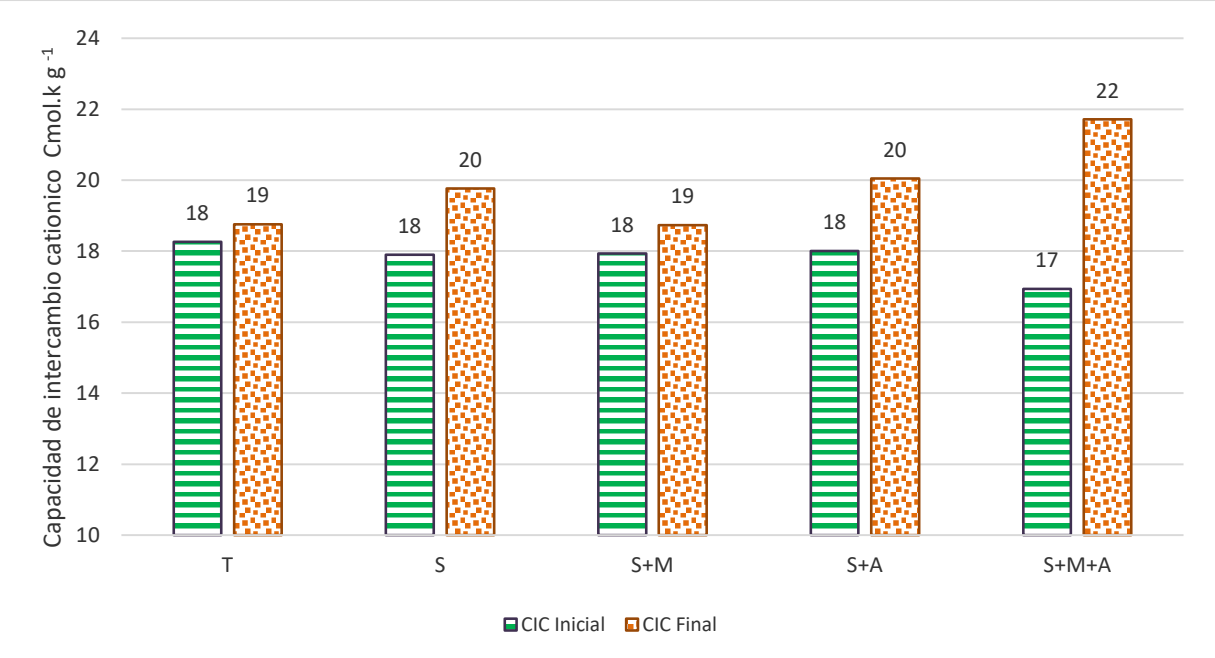

Vol. 2, Núm. 1 (2018) 
Figura 5. Cambio de la $\mathrm{CIC}\left(\mathrm{Cmol}^{+} \cdot \mathrm{kg}^{-1}\right)$ del suelo en diferentes tratamientos. Fuente: Autores

El efecto de las aplicaciones sobre la CIC presenta una tendencia al aumento en función del tiempo, lo que relaciona la importancia de compuestos orgánicos para la conservación de la fertilidad a través de sus cargas eléctricas y la dinámica de microorganismos como los que están presentes en el SUSPROT y el tratamiento con $\mathrm{S}+\mathrm{M}+\mathrm{A}$ (Figura 6). Esto se relaciona con lo citado por Sivapalan, en la cual reporta datos sobresalientes de fenoles asociados a la $\mathrm{CIC}$ de residuos agroindustriales y la capacidad de intercambio de materiales húmicos del suelo.

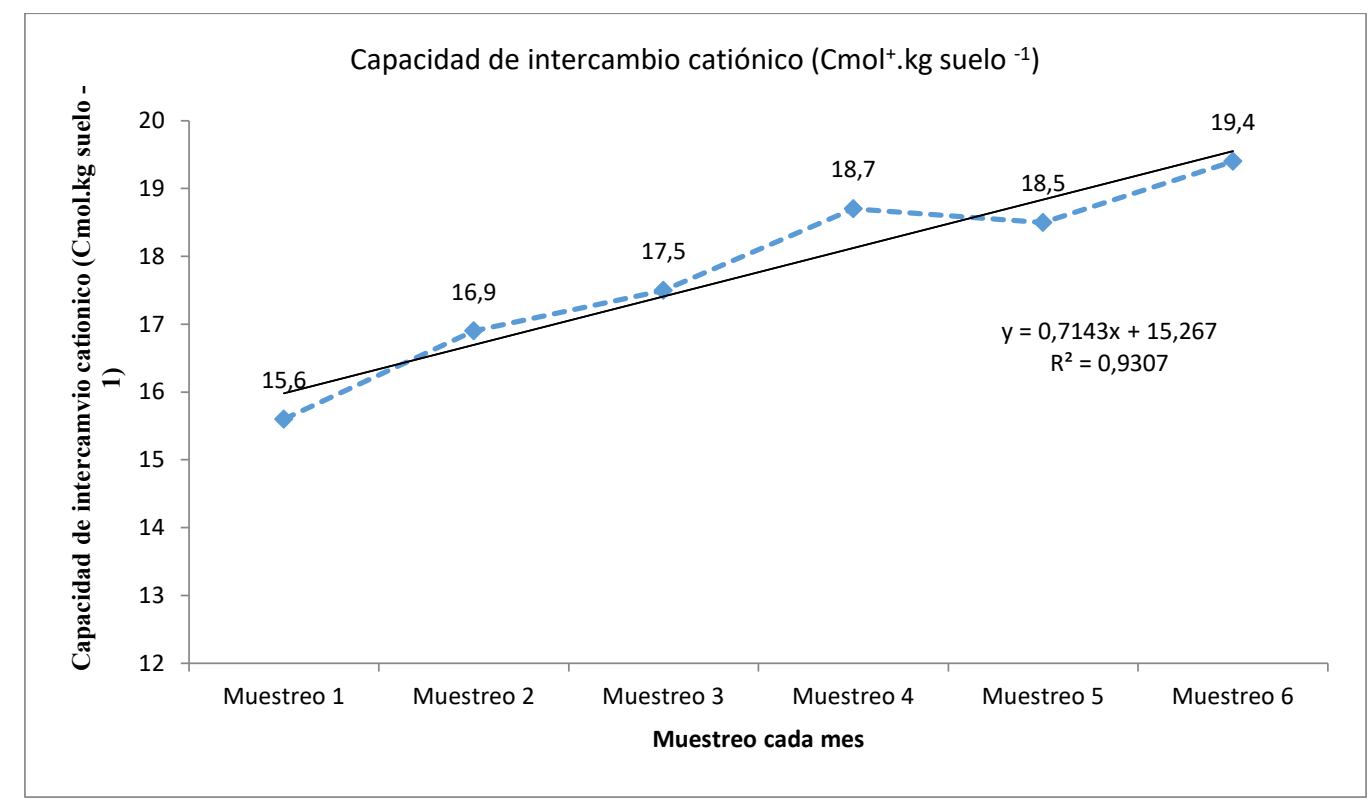

Figura 6. Cambio de la $\mathrm{CIC}(\mathrm{Cmol}+. \mathrm{kg}-1)$ del suelo en diferentes tratamientos. Fuente: Autores

Con relación a las limitaciones de oferta de nutrientes en este Inceptisol y al rendimiento del cultivo, el tratamiento "S" tuvo el mayor rendimiento (5.65 Ton/ha) (Figura 7), el cual obtuvo una diferencia significativa con relación a los otros tratamientos $(805 \mathrm{~kg} / \mathrm{ha}$ con el segundo el T3 que correspondió al S+M y $956 \mathrm{~kg} / \mathrm{ha}$ para el ultimo que fue el Testigo); Esta diferencia es importante desde la perspectiva administrativa y económica en términos de área de producción de biomasa fresca por hectárea. 


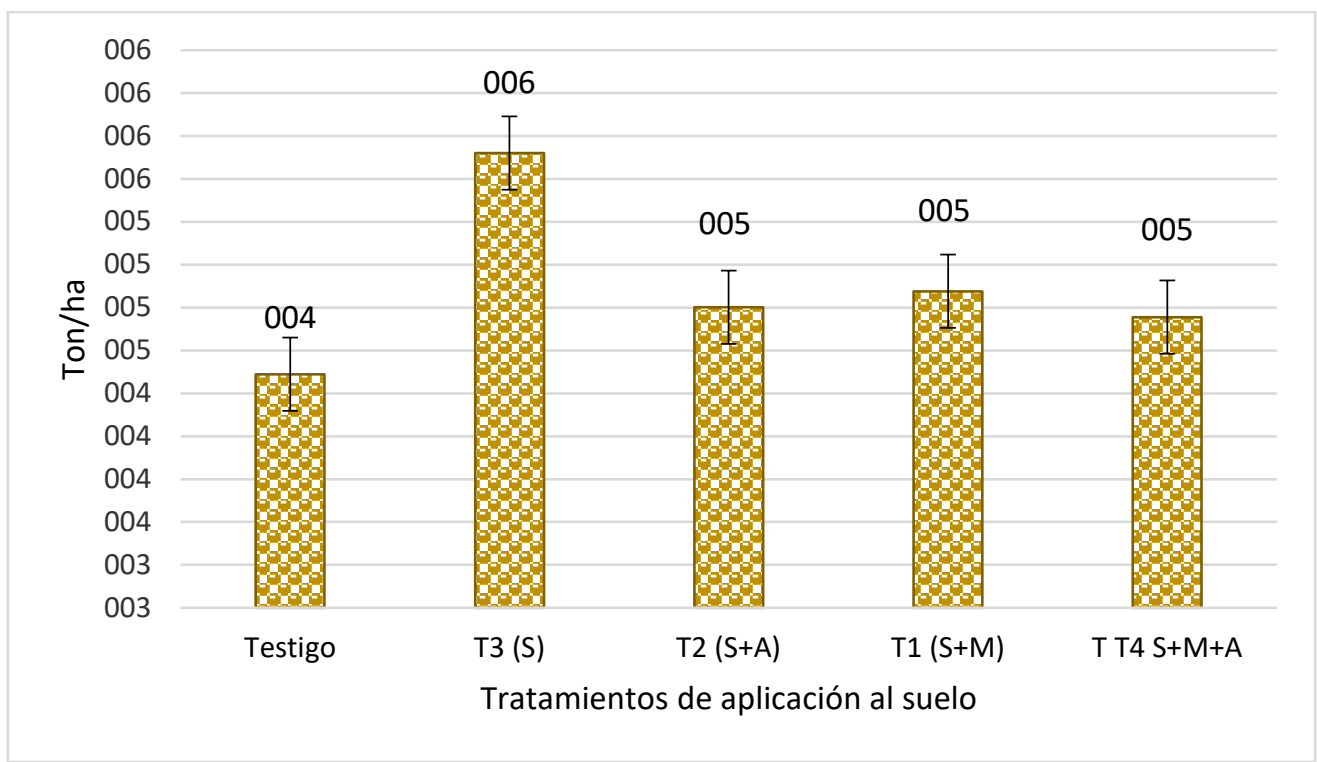

Figura 7. Rendimiento de Maíz (Zea mays) Hibrido 30k75. Fuente: Autores

La respuesta biológica del experimento estuvo en concordancia con los reportes Sanclemente et al. (2015), en los cuales registraron un aumento de valores de rendimiento del cultivo midiendo variables de interés económico por hectárea como la biomasa fresca.

\section{Conclusiones}

Las cantidades del fósforo encontradas después de las aplicaciones con SUSPORT, obedece un efecto positivo sobre el cultivo de maíz (Zea mays), considerando los tratamientos " $S$ " y " $S+M$ " con menores acumulaciones en el suelo y coincidiendo con los mayores rendimientos del cultivo $(5,65$ y 4,85 ton /ha respectivamente); esta apreciación se explica una alta correlación del fósforo foliar ( $\mathrm{R}^{2}$ : $0,92)$ donde el tratamiento "S+A" tuvo los mayores porcentajes de absorción foliar $(0.28 \%)$ seguido por el tratamiento "S", contrario con el Testigo $(\mathrm{T})$ que respondió con el menor contenido foliar, menor rendimiento y mayor acumulación de $\mathrm{P}$ en el suelo; por otra parte, el aumento de la CIC en todos los tratamientos con SUSPROT, se le considera importante la alta carga iónica contenida en los residuos aplicados. No obstante, en la variable Hierro, también se registró un aumento (más del $100 \%$ en algunos casos) después de las aplicaciones, incremento que puede ser atribuido a alto contenido de Hierro en el SUSPROT.

Se reconoce que la respuesta del Hibrido de Maíz (30k75) a la aplicación de este producto (SUSPROT) en cuanto al rendimiento y estrecha la relación con el contenido foliar de nutrientes principalmente en el tratamiento " $S$ ", que puede ser debido al aumento del contenido de elementos como $\mathrm{P}$, y Fe, y su posible disponibilidad en la absorción del suelo. En el Valle del Cauca, la evidencia experimental de este caso sugiere estudios más detallados que relacionen los comportamientos de estas propiedades químicas con estudio de la rizósfera del cultivo (actividad y biomasa microbiana y enzimas del suelo) para dar mejor respuesta a dichos efectos.

\section{Literatura citada}

Amaringo Villa, F. (2013). Determinación del punto de carga cero y punto isoeléctrico de dos residuos agrícolas y su aplicación en la remoción de colorantes. Revista de Investigación Agraria y Ambiental, 4(2), 27-36. doi:https://doi.org/10.22490/21456453.982

Ararat, M.C., Sinisterra, C. L. y Hernández, C. (2014). Valoraciones agronómicas y de rendimiento en la cosecha de "papa china" (Colocasia esculenta L.) en el trópico húmedo colombiano. Revista de Investigación Agraria y Ambiental, 5 (2), 169 - 180. 
Burbano O., H. (1994). La Materia Orgánica del Suelo en el Contexto de una Agricultura Sostenible. En: Sociedad Colombiana de la Ciencia del Suelo. Nueva Edición. Fertilidad de Suelos Diagnostico y Control. Santafé de Bogotá, D.C., Colombia. Ed. Silva M., Francisco, p.187-217.

Corredor Camargo, E., Fonseca Carreño, J., \& Páez Barón, E. (2012). Los servicios ecosistémicos de regulación: tendencias e impacto en el bienestar humano. Revista de Investigación Agraria y Ambiental, 3(1), 77-83. doi:https://doi.org/10.22490/21456453.936

Kiehl, E. J. (1985). Fertilizantes Orgânicos. Editora Agronômica "Ceres". Ltda. Sao Paulo. Brasil. $192 \mathrm{p}$.

Montaño, J. M. (2000). Respuesta de tres abonos orgánicos en cultivos de Rábano (Raphanus sativus L) y Lechuga (Latuca sativa L.), Tesis de pregrado Universidad Nacional de Colombia, Palmira.

Montenegro, S.P., Ararat, M.C., Betancur, J.F. (2015). Cachaza y carbonilla: residuos agroindustriales con potencial de fertilización biológica nitrogenada. Revista de Investigación Agraria y Ambiental, 6 (1), 83 - 89.

Peñaranda, L.V., Montenegro, S.P., Giraldo, P.A. (2017). Aprovechamiento de residuos agroindustriales en Colombia. Revista de Investigación Agraria y Ambiental, 8, (2), 141 150.

Ryan J., Singh B. (2015). Managing Fertilizers to Enhance Soil Health. First edition, IFA. International Fertilizer Industry Association 28, rue Marbeuf 75008 Paris, France.

Sanclemente, O.E., ARARAT, M. C., BALANTA, E. (2018). Evaluación preliminar de residuos sólidos en la Plaza de Mercado del municipio de Puerto Tejada (Cauca). Revista de Investigación Agraria y Ambiental, 9 (2). Recuperado de: http://hemeroteca.unad.edu.co/index.php/riaa/article/view/2349/2664

Sanclemente, O.E., Ararat, M.C. (2017). Consideraciones tecnológicas para el manejo agronómico sostenible de maíz en Colombia. Working Papers - ECAPMA. Recuperado de: http://hemeroteca.unad.edu.co/index.php/workpaper/article/view/1770

Sanclemente, O.E., Ararat, M.C., De la Cruz, C.A. (2015). Contribución de Vigna unguiculata L. a la sustentabilidad de sistemas de cultivo de caña de azúcar. Revista de Investigación Agraria y Ambiental, 6 (2), 47 - 56.

Sanclemente, O.E. (2015). Crisis de la agricultura convencional y agroecología como alternativa que aporta a la construcción de ciudadanía ambiental. En: Ciudadanía ambiental, crisis de la agricultura convencional y desafíos para una agroecología orientada hacia el desarrollo rural. Pág. 83 - 86. Universidad Nacional Abierta y a Distancia (UNAD). Recuperado de: http://hemeroteca.unad.edu.co/index.php/book/article/download/1324/1661

Sivapalan, K. (1981). Phenolic and exchange capacity of humic material. Soil Biol. Biochem. 13 (4), 331-333. 